\title{
Type-tuning of quasi-type-II CdSe/CdS seeded core/shell nanorods: type-I vs. type-II
}

\author{
Ahmet Fatih Cihan', Yusuf Kelestemur', Burak Guzelturk', \\ and Hilmi Volkan Demir ${ }^{1,2}$ \\ ${ }^{1}$ Department of Electrical and Electronics Engineering, Department of Physics, UNAM Institute of Materials Science and Nanotechnology, \\ Bilkent University, Ankara, 06800, Turkey \\ ${ }^{2}$ Microelectronics Division, School of Electrical and Electronics Engineering, and Physics and Applied Physics Division, School of Physical and \\ Mathematical Sciences, Nanyang Technological University, Nanyang Avenue, Singapore, 639798, Singapore \\ Email:volkan@bilkent.edu.tr and hvdemir@ntu.edu.sg
}

\begin{abstract}
We present tuning of quasi-type-II CdSe/CdS core/shell nanorods between type-I-like and type-II-like behavior in their amplified spontaneous emission pumped by 2-photon excitation, with the type attributions verified by time-resolved emission kinetics.

OCIS codes: (160.3380) Laser materials; (160.4236) Nanomaterials; (190.4180) Multiphoton processes; (300.6500) Spectroscopy, time-resolved.
\end{abstract}

Recent reports on the suppression of Auger recombination in semiconductor core/shell nanocrystals and nanorods (NRs) have made proper band engineering of their electronic structures essential. [1,2] One very promising platform for this kind of core/shell nanostructures is CdSe/CdS seeded core/shell NRs with quasi-type-II electronic structures. To date most optical gain studies on the core/shell nanostructures employed single-photon excitation, while two-photon absorption (TPA) is a strong alternative mainly because of lower photo-damage to the emitter with photons at the infrared wavelengths. In this regard, the core/shell NRs are most probably the best nanostructures with extremely high TPA cross-section, which is orders of magnitude larger than the alternative emitters for lasing applications. Very recently, a low threshold TPA-based amplified spontaneous emission (ASE) was demonstrated using the core/shell NRs. [3] Although the NRs have the potential to work in either type-I or typeII regimes [4], type-tuning of the ASE behavior of these NRs pumped by TPA mechanism has not been demonstrated, which is both scientifically and technologically important for fine-controlling the ASE peak. Here, we show that quasi-type-II CdSe/CdS NRs can be tuned from type-I-like to type-II-like behavior in their ASE based on TPA excitation, for which their type attributions are also confirmed by their independent time-resolved emission kinetics.
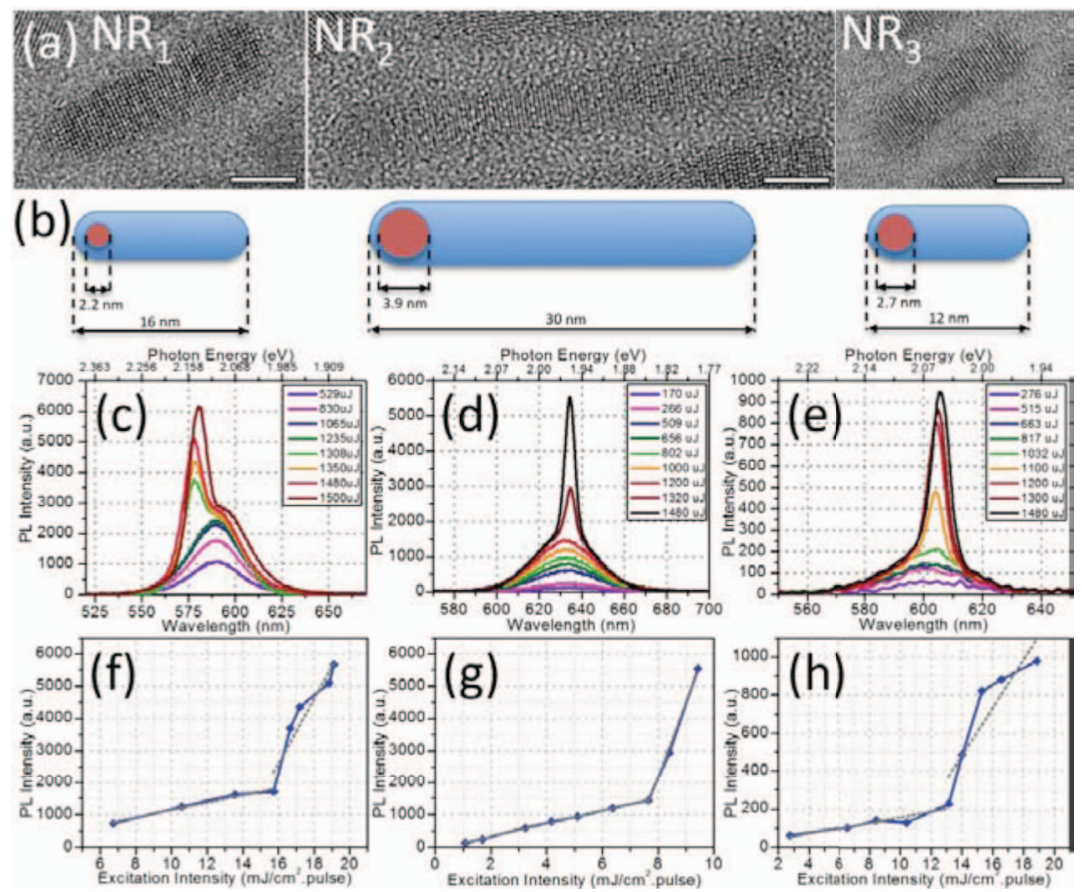

Fig. 1. (a) High resolution TEM images of three different NRs used in this study. (Size bars correspond to $5 \mathrm{~nm}$.) (b) Proportionally scaled illustration of core/shell sizes of the NRs. (c), (d), (e) Excitation intensity dependent emission spectra of $\mathrm{NR}_{1}$ (with blue-shifted ASE peak), NR 2 (with ASE peak at the same position as the spontaneous emission), and $\mathrm{NR}_{3}$ (with red-shifted ASE peak), respectively. (f), (g), (h) Excitation pulse intensity dependences of emissions at the ASE peak positions of $\mathrm{NR}_{1}, \mathrm{NR}_{2}$, and $\mathrm{NR}_{3}$, respectively [5]. 
In this work, we study exciton-exciton (X-X) interactions resulting in type-II to type-I operations from the same material system of $\mathrm{CdSe} / \mathrm{CdS} \mathrm{NRs}$ by adjusting their core/shell sizes. Here proper modifications of the core/shell dimensions result in repulsive $v s$. attractive $\mathrm{X}-\mathrm{X}$ interactions in the NRs, which in turn determines the spectral position of ASE with respect to the spontaneous emission (SE). For the experiments, close packed samples were prepared by drop-casting NR solutions $(100 \mathrm{mg} / \mathrm{mL})$ on quartz substrates and excited by $800 \mathrm{~nm}$ pulses with a 200 fs pulse width.

As presented in Figures 1(a)-(b), we used three NRs with different core/shell sizes to start with repulsive nature of the X-X interactions and modify it to the attractive one going from $\mathrm{NR}_{1}$ to $\mathrm{NR}_{3}$. The main reason behind the typetunability of this core/shell system is the shallow band offset (ca. $0.3 \mathrm{eV}$ ) between CdSe and CdS for electrons, unlike the higher offset for holes. Therefore, electrons in these structures are not necessarily confined in the core while holes are. Consequently, the $\mathrm{X}-\mathrm{X}$ interaction energy can be made positive or negative by modifying the electron wavefunction. For the case of very small core $\mathrm{NR}_{1}$, we observe a type-II behavior as expected because of the electron delocalization over the entire NR volume, which results in the blue-shifted ASE. Unlike $\mathrm{NR}_{1}, \mathrm{NR}_{3}$ exhibits red-shifting ASE behavior, which can be explained by the fact that the larger core of these NRs enables electron confinement in the core of the NRs making the X-X interaction attractive as observed in the type-I structures. For $\mathrm{NR}_{2}$ sample, however, the ASE takes place surprisingly almost at the same spectral position as the starting SE. This is because of the balance between the very long and thick shell and the large core of NRs resulting in neither attractive nor repulsive $\mathrm{X}-\mathrm{X}$ interaction.

To verify the electronic type assignments of these three NRs $\left(\mathrm{NR}_{1}\right.$ to type-II, $\mathrm{NR}_{2}$ to quasi-type-II, and NR 3 to type-I), we conducted time-resolved fluorescence (TRF) measurements (via time-correlated single photon counting) using low concentration in solution at the corresponding spectral emission peaks under low excitation intensity. For the type-II NRs, it is expected to have a longer single exciton decay lifetime compared to the type-I NRs because of the reduced electron and hole wavefunction overlap (overlap integral) and hence the reduced oscillator strength. Therefore, $\mathrm{NR}_{1}$ is expected to possess the longest lifetime, and $\mathrm{NR}_{3}$ to have the shortest lifetime. The results, as shown in Figure 2, are exactly in agreement supporting our type attributions inferred from the ASE peak positions.

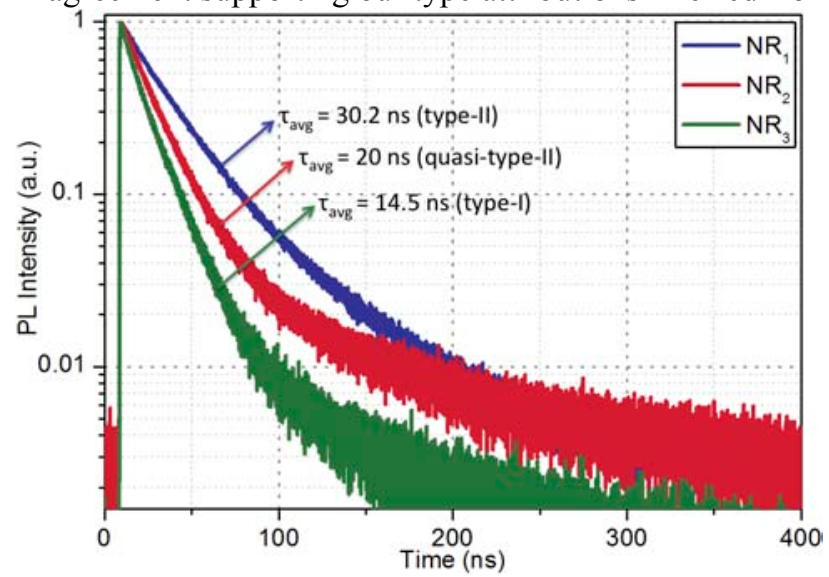

Fig. 2. TRF decay curves of the NRs together with average least chi-square fitting lifetimes [5].

In conclusion, we demonstrated type-tuning of $\mathrm{CdSe} / \mathrm{CdS}$ seeded core/shell nanorods in their amplified spontaneous emission pumped by two-photon excitation by engineering the nature of their X-X interactions. The type assignments from the ASE peak positions of these NRs were also verified by their independent TRF measurements in solution. In a specific set of $\mathrm{CdSe} / \mathrm{CdS} \mathrm{NRs}$ with the core/shell dimensions properly adjusted, the ASE peak exhibited no shift with respect to the starting SE. In this set of NRs, the X-X interactions is neither attractive nor repulsive, finely canceling each other out. With these findings, we conclude that, being suitable for electronic structure engineering, CdSe/CdS core/shell NRs are very promising candidates for TPA-based lasing studies.

This work is supported by National Research Foundation under Grant No. NRF-RF-2009-09 and NRF-CRP-6-2010-2, and also by EU-FP7 Nanophotonics4EnergyNoE, and TUBITAK EEEAG 109E002, 109E004, 110E010, 110E217, and 112E183. H.V.D. acknowledges support from ESF-EURYI and TUBA-GEBIP, A.F.C. Y.K., and B.G. from TUBITAK BIDEB.

\section{References}

[1] F. Garcia-Santamaria et al., Nano Letters, 9, 10, 3482-3488 (2009).

[2] Y. Louyer et al., Nano Letters, 11, 10, 4370-4375 (2011).

[3] G. Xing et al., ACS Nano, 6, 10835-10844 (2012).

[4] A. Sitt et al., Nano Letters, 9, 10, 3470-3476 (2009).

[5] A. F. Cihan et al., submitted. 